

\title{
Les ZNIEFF et les milieux forestiers
}

D. Richard, H. Maurin

\section{- To cite this version:}

D. Richard, H. Maurin. Les ZNIEFF et les milieux forestiers. Revue forestière française, 1991, 43 (S), pp.121-126. 10.4267/2042/26251 . hal-03425357

\section{HAL Id: hal-03425357 https://hal.science/hal-03425357}

Submitted on 10 Nov 2021

HAL is a multi-disciplinary open access archive for the deposit and dissemination of scientific research documents, whether they are published or not. The documents may come from teaching and research institutions in France or abroad, or from public or private research centers.
L'archive ouverte pluridisciplinaire HAL, est destinée au dépôt et à la diffusion de documents scientifiques de niveau recherche, publiés ou non, émanant des établissements d'enseignement et de recherche français ou étrangers, des laboratoires publics ou privés. 


\title{
Les ZNIEFF et les milieux forestiers
}

\author{
D. RICHARD - H. MAURIN
}

ZNIEFF : Zones naturelles d'Intérêt écologique, faunistique et floristique: l'acronyme (1) rebute, surprend, fait sourire, mais de toute façon, suscite la curiosité.

Le $Z$ pourrait rappeler de façon malheureuse les ZEP, ZAC, ZAD... qui, dans le langage de l'urbanisme, désignent une contrainte de zonage ; ce que, du point de vue juridique, les ZNIEFF ne sont pas.

II peut aussi évoquer le panneau d'indication routière: "Attention virage". Les ZNIEFF ont effectivement une vocation de mise en garde, de sonnette d'alarme. Le virage symbolise, lui, une nouvelle approche, une nouvelle direction de la part des administrations de l'environnement, mais aussi du monde des scientifiques et naturalistes, pour susciter une meilleure prise en compte du patrimoine naturel dans l'aménagement du territoire.

C'est ce symbole que nous avions retenu pour le titre du colloque organisé en mars 1990, sous l'égide de la Direction de la Protection de la Nature, au Muséum national d'Histoire naturelle, pour une présentation "grand public" des premiers résultats de l'inventaire. Ce titre, donc, "Les ZNIEFF, un virage à négocier, vers un réseau d'espaces naturels à gérer ", comporte un autre mot-clé, "négocier ". Les ZNIEFF se présentent en effet comme un outil de dialogue, au service des différents partenaires de l'aménagement du territoire, pour guider leurs décisions; cet outil a été réalisé à partir d'une synthèse des connaissances anciennes et récentes, fournies par des spécialistes, sur les milieux naturels, leur faune et leur flore remarquables.

Les actes de ce colloque ont tout récemment été publiés et sont disponibles au Secrétariat de la Faune et de la Flore.

\section{UN BREF RAPPEL}

\section{Le contexte}

L'inventaire ZNIEFF, qui concerne les milieux d'intérêt majeur, a débuté en 1982 et s'inscrit dans une troisième génération d'inventaires lancés par la Direction de la Protection de la Nature depuis une vingtaine d'années, avec le soutien scientifique et logistique du Secrétariat de la Faune et de la Flore, dans le cadre d'une convention-cadre ministère de l'Environnement/Muséum national d'Histoire naturelle.

La première génération concernait les inventaires d'espèces, en particulier Vertébrés, et en priorité les espèces menacées ou à problèmes. Ces inventaires ont donné lieu à la publication de divers atlas de répartition.

La deuxième génération correspondait à des inventaires thématiques par milieux: landes, dunes, tourbières, formations halophiles... II faut noter à ce sujet que les forêts n'ont jamais fait l'objet d'inventaires de ce type. Bien que constituant une synthèse de connaissances très précieuse, ces inventaires ont connu leurs limites, car les régions ont été inégalement couvertes, les méthodologies utilisées étaient hétérogènes...

Un des points forts de ZNIEFF, dans cette troisième génération, est sa relative exhaustivité sur l'ensemble du territoire métropolitain et, à terme, dans les départements d'Outre-Mer. Rappelons, pour mémoire, l'initiative pionnière du Centre technique du Génie rural, des Eaux et des Forêts, dans les années 1975, avec l'inventaire des richesses naturelles, dont les résultats ont constitué une base de travail importante pour les ZNIEFF.

(1) Acronyme: sigle prononçable comme un mot ordinaire (Petit Robert). 


\section{RICHARD - H. MAURIN}

\section{Les acteurs de l'inventaire}

Ils peuvent être classés en trois catégories:

- La trame scientifique et technique, composée de 22 comités scientifiques régionaux (plus les DOM), assistés dans la plupart des cas d'un secrétariat d'inventaire, et animant un réseau de collaborateurs, en grande partie bénévoles. En outre, le Secrétariat de la Faune et de la Flore assure le rôle de coordinateur de l'opération, au niveau national.

- La trame administrative, composée des 22 Délégations régionales à l'Architecture et à l'Environnement (DRAE), assurant le relais administratif du ministère de l'Environnement (Direction de la Protection de la Nature), commanditaire de l'inventaire et financeur principal.

- Les collectivités territoriales, principalement les Conseils régionaux, qui ont contribué au financement de l'inventaire dans plus de la moitié des régions, dans le cadre du $9^{\circ}$ plan.

II est important d'insister sur le caractère conjoncturel du premier type d'acteurs, à savoir les comités scientifiques et leur réseau de collaborateurs, qui ont été mobilisés à l'occasion de l'inventaire. À l'avenir, afin que l'inventaire soit actualisé en permanence, il est indispensable de pérenniser ces comités.

Une méthodologie standardisée au niveau national

Chaque comité scientifique a eu la tâche d'organiser la collecte des données, puis leur transcription selon un formulaire standard, mis au point par le Secrétariat de la Faune et de la Flore, en concertation avec scientifiques et administrations de l'environnement.

\section{- Rappelons la définition des ZNIEFF}

Une ZNIEFF est un secteur de territoire national, pour lequel les experts scientifiques ont identifié des éléments rares, remarquables, protégés ou menacés du patrimoine naturel (espèces animales, végétales ou associations d'espèces).

Dans l'inventaire, on distingue deux grands types de ZNIEFF :

- des zones de type II: grands ensembles riches et peu modifiés, ou qui offrent des potentialites biologiques importantes;

- des zones de type I: secteurs d'une superficie en général limitée, caractérisés par la présence d'espèces, ou d'associations d'espèces rares, remarquables, ou caractéristiques du patrimoine régional.

- Les informations recueillies sont de plusieurs types:

- des données administratives : région, département, communes concernées ;

- des données géographiques : altitude, surface, typologie, lithologie, mais surtout contour de la zone sur fonds cartographique de l'Institut géographique national au 1/25000 ou 1/50 000;

- des données biologiques: nomenclature phytosociologique, commentaire sur l'intérêt de la zone, bibliographie, et liste d'espèces.

La synthèse de ces données est organisée au Secrétariat de la Faune et de la Flore, à l'issue d'un traitement informatique comportant de nombreuses phases de validation.

Signalons dès maintenant que la diffusion des résultats de l'inventaire s'effectue à deux niveaux, conformément à la déontologie en vigueur :

- le niveau des informations de synthèse, c'est-à-dire caractéristiques administratives et géographiques, y compris contour de la zone, commentaire général sur l'intérêt de la zone. Ces données devraient faire l'objet d'une large diffusion auprès des administrations et des collectivités territoriales ;

- le niveau des informations brutes, c'est-à-dire les listes d'espèces, dont la diffusion est subordonnée à l'accord du Comité scientifique.

Si la méthodologie était standard, on ne pouvait toutefois, dans le cadre de ce programme qui a mobilisé, pour la première fois, un réseau de collaborateurs très divers (sociétés savantes, muséums, laboratoires universitaires, naturalistes amateurs, parfois aussi forestiers et personnels de l'Office 
national de la Chasse...), obtenir un produit irréprochable dès la première " remise de copie ". Le bilan national tiré à l'occasion du colloque de mars a en effet montré certaines lacunes dans l'inventaire, ainsi que quelques incohérences.

La deuxième phase de l'inventaire consiste notamment à compléter et harmoniser ces résultats. La mise à jour des donnèes, en fonction des connaissances nouvelles et de l'évolution des milieux, est également un objectif essentiel des prochaines phases de l'inventaire.

\section{LES RÉSULTATS}

Au mois de mars 1990, 13344 ZNIEFF étaient recensées en métropole, ce qui concerne environ $50 \%$ des communes. Dans les DOM, l'inventaire est moins avancé, pour des raisons d'organisation et de financement, mais la dynamique est maintenant relancée, en particulier en Guyane, et l'on peut attendre des résultats interessants pour la fin 1991.

\begin{tabular}{|c|c|c|c|c|}
\hline Régions & $\begin{array}{l}\text { Nombre de } \\
\text { départements }\end{array}$ & $\begin{array}{c}\text { Nombre } \\
\text { de ZNIEFF }\end{array}$ & Zone type 1 & Zone type II \\
\hline He-de-France & 8 & 676 & 602 & 74 \\
\hline Champagne-Ardenne $\ldots \ldots \ldots \ldots \ldots$ & 4 & 530 & 475 & 55 \\
\hline Picardie . . . . . . . . . . . . . & 3 & 473 & 384 & 89 \\
\hline Haute-Normandie . .............. & 2 & 453 & 384 & 69 \\
\hline Centre $\ldots \ldots \ldots \ldots \ldots \ldots \ldots \ldots$ & 6 & 779 & 647 & 132 \\
\hline Basse-Normandie $\ldots \ldots \ldots \ldots \ldots \ldots$ & 3 & 411 & 351 & 60 \\
\hline Bourgogne $\ldots \ldots \ldots \ldots \ldots \ldots \ldots$ & 4 & 406 & 211 & 195 \\
\hline Nord-Pas-de-Calais . ............. & 2 & 308 & 281 & 27 \\
\hline Lorraine $\ldots \ldots \ldots \ldots \ldots \ldots$ & 4 & 558 & 540 & 18 \\
\hline Alsace $\ldots \ldots \ldots \ldots \ldots \ldots \ldots \ldots \ldots$ & 2 & 247 & 203 & 44 \\
\hline Franche-Comté $\ldots \ldots \ldots \ldots \ldots \ldots$ & 4 & 664 & 594 & 70 \\
\hline Pays-de-la-Loire . . . . . . . . . . . & 5 & 580 & 426 & 154 \\
\hline Bretagne $\ldots \ldots \ldots \ldots \ldots \ldots \ldots$ & 4 & 559 & 482 & 77 \\
\hline Poitou-Charentes ............... & 4 & 612 & 580 & 32 \\
\hline Aquitaine $\ldots \ldots \ldots \ldots \ldots \ldots$ & 5 & 433 & 138 & 295 \\
\hline Midi-Pyrénées $\ldots \ldots \ldots \ldots \ldots \ldots \ldots$ & 8 & 1440 & 1309 & 131 \\
\hline Limousin .... . & 3 & 240 & 153 & 87 \\
\hline Rhône-Alpes ................. & 8 & 1952 & 1788 & 164 \\
\hline Auvergne $\ldots \ldots \ldots \ldots \ldots \ldots \ldots$ & 4 & 372 & 345 & 27 \\
\hline Languedoc-Roussillon & 5 & 952 & 706 & 246 \\
\hline Corse $\ldots \ldots \ldots \ldots \ldots \ldots \ldots \ldots \ldots$ & 2 & 224 & 183 & 41 \\
\hline Provence-Alpes-Côte d'Azur ......... & 6 & 475 & 397 & 78 \\
\hline Total France métropolitaine .......... & 96 & 13344 & 11179 & 2165 \\
\hline Superficies correspondantes .. & $553000 \mathrm{~km}^{2}$ & $123000 \mathrm{~km}^{2}$ & $42000 \mathrm{~km}^{2}$ & $107000 \mathrm{~km}^{2}$ \\
\hline
\end{tabular}

L'inventaire se poursuit dans les DOM où l'on a recensé :

\begin{tabular}{|c|c|c|c|}
\hline Département & Nombre de ZNIEFF & Zone type 1 & Zone type II \\
\hline Réunion & 45 & 33 & 12 \\
\hline Martinique $\ldots \ldots \ldots \ldots \ldots$ & 3 & 2 & 1 \\
\hline Guadeloupe ............. & 3 & 3 & 0 \\
\hline
\end{tabular}




\section{RICHARD - H. MAURIN}

\section{LES ZNIEFF APPLIQUÉES AUX FORÊTS}

Sur les 13344 ZNIEFF métropolitaines, 3573 ont été répertoriées, avec comme typologie principale " forêt "; dont 2814 en zones de type I et 759 en zones de type II, cela représente $27 \%$ de l'ensemble des ZNIEFF, soit le type de milieu le plus retenu avant "lac, réservoir, étang" $=12 \%$ et "marais, tourbière $"=11 \%$. Dans certaines régions, comme la Réunion, deux tiers des ZNIEFF sont forestières.

II est à noter que bien d'autres milieux concernent également les périmètres d'intervention des forestiers, comme les tourbières, les landes ou même certaines pelouses sèches.

II apparaît que ZNIEFF ne peut constituer une réponse standard aux problèmes de gestion que se posent les forestiers. Comment le pourrait-il d'ailleurs lorsque tant de questions concernant la recherche fondamentale sur le fonctionnement des écosystèmes forestiers restent posées ?

ZNiEFF constitue par contre un bon répertoire des principaux enjeux de conservation du patrimoine naturel forestier; qu'il s'agisse de la présence d'espèces animales ou végétales en populations relictuelles, de groupements végétaux spécialisés liés à un microclimat ou une géomorphologie particulière, ou encore de formations représentatives d'un climax climatique.

II est vrai qu'un des problèmes-clés est celui de la disponibilité de l'information pour le gestionnaire. Dans ce sens, une importante réflexion doit être menée entre scientifiques et gestionnaires pour :

- considérer l'opportunité de révéler les stations d'espèces rares forestières. Cela suppose évidemment l'établissement de relations de confiance, qui passent peut-être par l'établissement d'une charte d'utilisation des ZNIEFF par les forestiers ;

- examiner au cas par cas les problématiques de la conservation. Comme cela a été souvent évoqué, la protection d'une station de plantes rares ne signifie pas nécessairement non gestion ;

- étudier les moyens et les conditions d'une consultation, à intervalles réguliers ou au coup par coup du Comité scientifique, par les forestiers. Cela suppose, bien sûr, une pérennisation de ce Comité scientifique ;

- envisager l'apport des connaissances acquises par les forestiers, non encore intégrées à ZNIEFF, notamment à travers la description des stations typologiques forestières.

\section{LA DIMENSION INTERNATIONALE}

\section{Les inventaires européens}

- La Communauté économique européenne a lancé, en 1983, en liaison avec le Conseil de l'Europe, un vaste programme d'inventaire des données de l'environnement, le programme CORINE (Coordination de I'Information en Environnement). Un des thèmes abordés est l'inventaire des milieux naturels et semi-naturels les plus remarquables dans la Communauté ; il s'agit de CORINE-Biotopes, très proche dans sa finalité de l'inventaire ZNIEFF.

Dans le cadre de ce programme, une typologie des milieux présents dans la Communauté a été établie. Elle comporte plusieurs versions successives qui intègrent au fur et à mesure les connaissances fournies par les différents pays membres. La dernière version, qui devrait inclure de nombreux compléments fournis par la France, en particulier pour les espaces supra-forestiers, est en cours d'achèvement.

À terme, cette typologie européenne, adaptée et complétée, devrait constituer une référence pour la description de tous les espaces protégés français.

- Cette même typologie sert de base à la définition de milieux recensés dans l'annexe 1 de la future Directive Habitats, en cours de discussion au niveau européen.

II s'agit cette fois d'un projet de texte réglementaire, à l'instar de la Directive Oiseaux de 1979, qui engagera formellement les pays membres à une protection effective de certains des biotopes mentionnés dans cette annexe 1 (les annexes 2, 5 et 6 concernent quant à elles des espèces à protéger). 
Pour les forêts, sont prises en considération "les forêts (sub)naturelles d'essences indigènes et spontanées dans la station, existant à l'état de futaies avec sous-bois typique, et répondant aux critères suivants :

- rares et résiduelles dans leur aire potentielle;

- hébergeant des essences endémiques ou menacées;

- à rôle important comme forêts de protection".

Différents types retenus concernent la France :

\section{Forêts de l'Europe tempérée}

41.11 Hêtraies du Luzulo-Fagetum

41.12 Hêtraies à Houx, Taxus, riches en épiphytes

41.13 Hêtraies de l'Asperulo-Fagetum

41.14 Hêtraies subalpines à Erable et Rumex arifolius (forêts de protection)

41.15 Hêtraies calcicoles du Cephalenthero-Fagion

41.24 Chênaies du Stellario-Carpinetum

41.26 Chênaies du Galio-Carpinetum

41.4 Forêts de ravins du Tilio-Acerion

44.3 Forêts alluviales résiduelles

44.A Tourbières boisées

Forêts méditerranéennes à feuilles caduques

41.86 Frênaies à Fraxinus angustifolia

44.6 Forêts-galeries à Salix alba et Populus alba

Forêts sclérophylles méditerranéennes endémiques ou résiduelles

45.1 Forêts à Olea et Ceratonia

45.2 Forêts à Quercus suber

45.3 Forêts à Quercus ilex

Forêts de Conifères

- alpines et subalpines

42.2 Forêts acidophiles du Vaccinio-Picetea

42.3 Forêts à Mélèze et Pin cembro des Alpes (forêts de protection)

42.4 Forêts de Pin à crochets des Pyrénées et Alpes occidentales

- méditerranéennes montagnardes

42.6 Pinèdes méditerranéennes de Pins noirs endémiques

42.8 Pinèdes méditerranéennes de Pins mésogéens endémiques

42.A7 Forêts à Taxus baccata

42.A Forêts à Juniperus thurifera

\section{L'apport de l'inventaire ZNIEFF}

Nous concluerons notre article avec un exemple, qui illustre le rôle que joue dès maintenant et qu'aura à jouer l'inventaire ZNIEFF dans l'avenir, pour orienter des choix de décision tant aux niveaux régional que national et international.

Devant l'importance des enjeux de la future Directive Habitats, le ministère de l'Agriculture s'est associé au ministère de l'Environnement pour confier au Secrétariat de la Faune et de la Flore une exploitation de l'ensemble du fichier ZNIEFF, visant à déterminer la répartition et les sites les plus intéressants pour :

- les espèces de faune et de flore mentionnées dans l'annexe 1 de la future Directive (espèces dont l'habitat est menacé dans la Communauté) ;

- les biotopes mentionnés dans l'annexe 1 de cette même Directive.

Les résultats de cette étude devraient permettre d'évaluer le champ d'application de la future Directive sur le territoire français et de sélectionner, sur des bases rationnelles, en concertation avec les régions concernées, les zones qui pourront être désignées par la France auprès de la Communauté. 


\section{PICHARD - H. MAURIN}

Les administrations de l'État voient ainsi en ZNIEFF — et les études qui pourront être menées sur leurs bases - des moyens de justifier, vis-à-vis de la Communauté internationale, la propre approche de la France quant à la constitution des réseaux de zones protégées.

D. RICHARD - H. MAURIN

Secretariat de la Faune et de la Flore MUSÉUM NATIONAL D'HISTOIRE NATURELLE

57, rue Cuvier

75231 PARIS CEDEX 